www.jmscr.igmpublication.org

Impact Factor 5.84

Index Copernicus Value: 71.58

ISSN (e)-2347-176x ISSN (p) 2455-0450

crossref DOI: _https://dx.doi.org/10.18535/jmscr/v5i10.114

Journal Of Medical Science And Clinical Research

IGM Publication

An Official Publication of IGM Publication

\title{
A Comparative Study for Control of Shivering with Clonidine, Butorphanol and Tramadol in Patients Undergoing Neuraxial Blockade
}

\author{
Authors \\ Dr Somnath Longani ${ }^{1}$, Dr Abhishek Srivastava ${ }^{2}$, Dr Jaishree Bogra ${ }^{3}$, \\ Dr Archana Agarwal ${ }^{4}$ \\ ${ }^{1}$ Associate Professor, Department of Anesthesiology, Critical Care and Pain Medicine, Hind Institute of \\ Medical Sciences, Barabanki, Uttar Pradesh, India \\ ${ }^{2}$ Assistant Professor, Department of Anesthesiology, Critical Care and Pain Medicine, Hind Institute of \\ Medical Sciences, Barabanki, Uttar Pradesh, India \\ ${ }^{3,4}$ Professor, Department of Anesthesiology, Critical Care And Pain Medicine, Hind Institute of Medical \\ Sciences, Barabanki, Uttar Pradesh, India \\ Corresponding Author \\ Dr Abhishek Srivastava
}

Assistant Professor, Department of Anesthesiology, Critical Care and Pain Medicine, Hind Institute of

Medical Sciences, Barabanki, Uttar Pradesh, India

\section{Abstract}

Background: Shivering is a typical issue amid neuraxial anesthesia. This investigation intended to assess the relative adequacy of intravenously directed clonidine, butorphanol and tramadol for control of intraoperative shivering in patients undergoing Neuraxial Blockade.

Materials and Methods: This investigation was led in 120 patients of age 25-50 years. Neuraxial block was performed with $2.8 \mathrm{~mL}(14 \mathrm{mg})$ of $0.5 \%$ bupivacaine overwhelming in all patients. On shivering, patients were arbitrarily assigned to get an intravenous, $1 \mathrm{~mL}$ bolus dosage of $50 \mathrm{mg}$ tramadol, or $1 \mathrm{mg}$ butorphanol, or $150 \mu \mathrm{g}$ clonidine, in a twofold blinded way. Control of shivering, time taken for discontinuance, recurrence, hemodynamic changes, axillary temperatures, and side effects were noted and analyzed for each of the 3 gatherings. Shivering was reviewed from 0 to 4 grades and, if review 3 shivering happened, the investigation medicate was considered as inadequate and intravenous pethidine $25 \mathrm{mg}$ was given as safeguard sedate.

Result: Time taken to control Shivering was altogether lower in Group I (Tramadol) when contrasted with Group II (Butorphanol). More patients with higher sedation score with Butorphanol amass contrasted with Tramadol Group. Nausea and regurgitating higher in Tramadol Group contrasted with Butorphanol Group.

Conclusion: Tramadol is most fast acting and successful responsible for control of shivering with neauraxial block with no critical symptoms and minimum return of shivering when contrasted with Butorphanol and Clonidine.

Keywords: Shivering; Spinal anaesthesia; Clonidine; Tramadol.

\section{Introduction}

Shivering is upsetting for the patients experiencing surgery under both local and after general anesthesia. The fundamental driver for shivering intra/postoperatively are temperature misfortune, diminished thoughtful tone and 
foundational arrival of pyrogens. ${ }^{1}$ Shivering expands use of heart and foundational vitality, bringing about expanded oxygen utilization and carbon dioxide creation, lactic acidosis and raises the intraocular and intracranial weight. It likewise meddles with haemodynamic checking intraoperatively. ${ }^{2}$

In spite of the fact that the component of root of shivering is not clear, different theories have been proposed to clarify its event. Perioperative hypothermia is the essential driver, which happens due to neuraxial anaesthesia- initiated restraint of thermoregulatory instrument. Shivering happens as a thermoregulatory reaction to hypothermia or muscle action with tonic or clonic examples, and different frequencies have been noticed. ${ }^{3}$ However, in the postoperative period, muscle movement might be expanded even with normothermia, recommending that systems other than warm misfortune with consequent reduction in the center temperature add to the starting point of shivering. These might be uninhibited spinal reflexes, thoughtful over-movement, postoperative agony, adrenal concealment, pyrogen discharge and respiratory alkalosis. ${ }^{4}$ Due to shivering and warm discomfort, the nature of patient recuperation endures.

Different techniques are accessible to control shuddering amid anesthesia, which incorporate non-pharmacological strategies (like covering the patient with blankets, utilization of brilliant warmth and warming the working room, utilization of warm local sedative arrangement or warm intravenous liquids) and pharmacological strategies utilizing drugs like opioids (pethidine, nalbuphine, or tramadol), ketanserin, propofol, granisetron, doxapram, physostigmine, clonidine, and nefopam, however banter on a 'perfect against shuddering medication' proceeds. ${ }^{5}$

Tramadol hydrochloride, a $\mu$-opioid receptor agonistic medication, has a modulatory impact on central mono-aminergic pathways, and consequently restrains the neuronal take-up of noradrenaline/serotonin and empowers hydroxytriptamine discharge which resets the body temperature control focus. It has picked up a notoriety in numerous clinical trials for the control of shivgering. ${ }^{6}$ The counter shuddering impact of butorphanol is likewise $\kappa$-opioid receptor intervened. The impact of opioids on body temperature and thermoregulatory reaction interceded through their activity on preoptic front hypothalamus neurons, dorsal raphe core neurons, raphe magnus neurons and spinal cord. By and large, opioids exerts a variety of stimulus effects on signal transduction. They increment development of cyclic adenosine monophsphate which increases thermo-affectability in warm-sensitive and moderate-slope temperature-insensitive neurons. ${ }^{7}$ Clonidine, an alpha-2 agonist sedate, diminishes the arrival of noradrenaline from the axonal terminals in the hypothalamus to apply its against shivering impacts. This clinical trial was embarked to think about the viability of clonidine, butorphanol, and tramadol for controlling perioperative shivering of surgical patients experiencing Neuraxial Blockade.

\section{Materials and Methods}

This investigation was directed in hospital in U.P, in division of Anesthesiology. In the wake of getting endorsement of the morals board of trustees and acquiring composed educated assent from patients, 120 patients of either sex, matured between 25 to 50years, planned for elective lower stomach and lower appendage surgeries, under spinal anesthesia, who created intraoperative shivering post spinal anesthesia of grade 3 or 4 going on for least time of 2 minutes were incorporated into this forthcoming randomized clinical controlled examination. Patients with history of fever, known affectability to medications to be utilized, shivering even before overseeing spinal anesthesia, prerequisite of supplementation with general anesthesia were barred from the examination.

The cases were arbitrarily apportioned to one of 3 groups by a specialist aiming to set up the examined medicate arrangement: Group I got an intravenous bolus of $50 \mathrm{mg}(1 \mathrm{~mL})$ tramadol; 
Group II got an iv bolus of $1 \mathrm{mg}(1 \mathrm{~mL})$ butorphanol and Group III got an iv bolus of 150 $\mu \mathrm{g}(1 \mathrm{~mL})$ clonidine. Further interventions and monitoring were done by an investigator blinded to the group allocation.

Patients were assessed preoperatively and inj. Glycopyrolate $0.2 \mathrm{mg}$ IV was given as a premedication. Preloading of liquid was done with one liter of warm Ringer lactate. Screens were joined and benchmark vitals were recorded when persistent was taken into Operation Theater. Spinal anesthesia was founded at L3-L4 or L2-L3 interspace in sitting position with spinal needle no.25G, Bupivacaine substantial $0.5 \%$ was utilized for spinal anesthesia. Surgery was begun after accomplishment of the satisfactory level of sensory and motor block.

Oxygen was regulated to all the patients of the three gatherings at a rate of $5 \mathrm{~L} / \mathrm{min}$ with confront veil, and patients were secured with drapes yet not effectively warmed. No methods for dynamic rewarming were utilized. Intravenous liquids and analgesic medications were directed at room temperature.

Standard checking of heartbeat rate was done, pulse, oxygen saturation (SPO2), body temperature (axillary) were recorded before the initiation of surgery and from that point at regular intervals from the standard for 60 minutes; and like clockwork, for whatever is left of the perception time frame.

Grading of shivering was done according to Wrench,6 which is as per the following:
Grade 0: No shivering

Grade 1: One or more of the accompanying: Piloerection, Peripheral vasoconstriction, peripheral cyanosis with, yet without obvious muscle action

Grade 2: Visible muscle activity confined to one muscle group

Grade 3: Visible muscle activity in more than one muscle group

Grade 4: Gross muscle activity involving the whole body

Patients who grew either group 3 or 4 of shivering were incorporated into the study.Side effects like nausea, regurgitating, bradycardia, hypotension, unsteadiness; and sedation score were recorded.

\section{Result}

Time taken to control Shivering was altogether lower in Group I (Tramadol) when contrasted with Group II and III. More patients with higher sedation score with Butorphanol amass contrasted with Tramadol Group. Nausea and regurgitating higher in Tramadol Group.

Butorphanol and tramadol were more effective than clonidine in suppressing shivering.

Butorphanol, tramadol, and clonidine completely controlled rigors in $81 \%, 75 \%$,

and $52 \%$ of cases, respectively. Time taken to terminate rigors was significantly higher for clonidine (3.1 \pm 1 minutes) than for butorphanol and tramadol $(2.3 \pm 0.7$ minutes and $1.9 \pm 0.8$ minutes)

Table 1: Demographic profile

\begin{tabular}{|l|c|c|c|}
\hline Variable & Group I $(\mathrm{n}=40)$ & Group II $(\mathrm{n}=40)$ & Group III $(\mathrm{n}=40)$ \\
\hline Age (years) & $35.7 \pm 5.7$ & $36.8 \pm 4.8$ & $39.7 \pm 7.4$ \\
\hline Gender & & & 22 \\
Male & 26 & 18 & 13 \\
Female & 14 & $56.3 \pm 5.4$ & $59.4 \pm 6.3$ \\
\hline Weight $(\mathrm{kg})$ & $57.9 \pm 4.3$ & $162.3 . \pm 4.7$ & $164.7 \pm 4.9$ \\
\hline Height $(\mathrm{cm})$ & $163.7 \pm 6.2$ & $67.4 \pm 21.5$ & $69.7 \pm 18.8$ \\
\hline Duration of surgery (mins) & $66.7 \pm 17.4$ & $126.7 \pm 14.6$ & $127.6 \pm 13.3$ \\
\hline Duration of spinal block (mins) & $128.4 \pm 15.4$ & & \\
\hline
\end{tabular}


Table 2: Shivering response

\begin{tabular}{|l|c|c|c|}
\hline Parameters & Group I $(\mathrm{n}=40)$ & Group II $(\mathrm{n}=40)$ & Group III $(\mathrm{n}=40)$ \\
\hline Control of shivering & & & \\
Complete & 30 & 32 & 21 \\
Incomplete & 10 & 8 & 19 \\
\hline Time taken for control of shivering & $1.9 \pm 0.8$ & $2.3 \pm 0.7$ & $3.1 \pm 1.0$ \\
\hline Recurrence of shivering & 3 & 5 & 9 \\
\hline
\end{tabular}

Table 3: Side effects

\begin{tabular}{|l|c|c|c|}
\hline Complications & Group I & Group II & Group III \\
\hline Nausea & 4 & 0 & 0 \\
\hline Vomiting & 1 & 0 & 0 \\
\hline Hypotension & 0 & 0 & 2 \\
\hline Bradycardia & 0 & 0 & 4 \\
\hline Dry mouth & 2 & 0 & 1 \\
\hline
\end{tabular}

\section{Discussion}

Our investigation has demonstrated that prophylactic utilization of Butorphanol, clonidine and tramadol were powerful in avoiding shivering amid neuraxial anesthesia. Tramadol is a midway acting pain relieving that has powerless opioid agonist properties. It likewise restrains serotonin and norepinephrine take-up in the spinal cord and is effective in the treatment of post-agent shivering after regional and general anaesthesia. ${ }^{8}$ Tramadol may cause nausea and vomiting. Tramadol has low risk of respiratory sorrow, resistance and reliance. Clonidine is a centrally acting $\alpha 2$, agonist and the anatomic focus of its hostile to shivering impact can be found at three levels. It diminishes the thermoregulatory edge for vasoconstriction and shivering as the hypothalamus contains high-thickness a2 receptors. It likewise decreases unconstrained terminating in locus coeruleus - a pre-shivering focus in the pons. The statistic profile and the middle level of sensory blockade in all the three gatherings were similar.

Regional anesthesia, either focal neuraxial block or peripheral nerve block, is a safe and extremely prevalent method utilized for different surgeries. In any case, $40 \%$ to $70 \%$ of patients experiencing regional anesthesia create shivering, however it is likewise found to happen after general anesthesia. ${ }^{9}$ In the present examination, the components that impact the event of shivering, similar to temperature of IV liquids and medications, were not firmly controlled, but rather this ought not influence the legitimacy of our investigation on the grounds that the present investigation is centered around reaction to treatment utilized as opposed to frequency of shivering; and by randomization, each of the three gatherings were subjected to comparable degrees of impact of these elements.

Tramadol and butorphanol both had equivalent outcomes in entire concealment of shivering which agrees with perceptions made by Atashkhoyi and Negargar, ${ }^{11}$ Dhimar et al, ${ }^{12}$ and Bhatnagar et al. ${ }^{13} \mathrm{~A}$ higher frequency of repeat of rigors was seen in clonodine - treated patients in our examination, which is like perceptions made by Maheshwari et $\mathrm{al}^{10}$. Clonidine-treated patients had a lower adequacy responsible for shivering, with equivalently higher recurrence than patients in the other 2 gatherings. This finding is in inconsistency with the investigation done by Schwarzkopf et $\mathrm{al}^{14}$ and Horn et $\mathrm{al}^{15}$ who noted $100 \%$ response rates in charge or counteractive action of rigors with clonidine after general anesthesia, however these creators additionally watched repeats with clonidine. The time taken for control of shuddering was additionally essentially higher with the clonidine group than with the tramadol and butorphanol gatherings.

We have discovered that Tramadol has controlled shivering in $1.9 \pm 0.8 \mathrm{~min}$ which is practically identical to finding of Chen S C et al. ${ }^{16}$ This is speedier than Clonidine which is $3.1 \pm 1.0$ 
moment in our investigation which is as opposed to think about by Parvin Sajedi et $\mathrm{al}^{17}$ who have announced that Clonidine and Tramadol have comparable impact in speed and control of shivering. Zehedi et al ${ }^{18}$ have additionally detailed speedier activity of Tramadol than Clonidine, in spite of the fact that there mean time was 5 min and 9 min individually. The speed of action of Butorphanol in our examination is $2.3 \pm 0.7 \mathrm{~min}$ which is factually critical slower than Tramadol and is measurably speedier than Clonidine. Vogelsang $\mathrm{J}$ et $\mathrm{al}^{19}$ has reported time of 2-5 min with Butorphanol which is like our finding. In our examination, we have discovered that Tramadol has quickest beginning of activity of against shivering impact when contrasted with Butorphanol and Clonidine.

The complications were observed to be higher in case of tramadol contrasted with clonidine and butorphanol. In the present investigation, the rate of nausea was higher in tramadol gathering contrasted with other two gatherings. Comparable contrasts were noted between the two gatherings in connection to vomiting and dizziness. Studies led by Gangopadhyay et al $1^{20}$ announced a higher frequency of vomiting with tramadol than clonidine and butorphanol, while Maheshwari et $\mathrm{al}^{10}$ demonstrated a higher occurrence of vomiting with butorphanol contrasted and tramadol. The literature supports higher rate of emesis with opioids, however the dosages utilized by us were rarely connected with this antagonistic impact. It is additionally outstanding that repeated dosing of tramadol is related with a high rate of emesis, butorphanol with sedation and respiratory depression, and clonidine with hypotension and drowsiness. The frequency of these side effects was higher in different examinations after repeated drug dosing to abolish rigors, which may clarify the explanation behind their lower occurrence in our study. ${ }^{21}$

A constraint of this examination is that we couldn't gauge the core body temperature. For estimation of core body temperature, the test should be placed in the throat or close to the tympanic membrane. Both these are awkward and unsatisfactory who has been given spinal anesthesia. Rectal temperature checking was a probability yet was not attempted.

\section{Conclusion}

We conclude that butorphanol and tramadol are better than clonidine for administration of postoperative shivering because of higher rates of accomplishment, prior beginning of activity and less recurrence with equivalent levels of security. At present opioids hold a high reputation as reliable anti-shivering agents, however the look for a perfect substitute still proceeds.

\section{References}

1. Sessler DI, Ponte J. Shivering during epidural anesthesia. Anesthesiology 1990;72:816-21.

2. Pascal A. Postanesthetic Shivering: Epidemiology, Pathophysiology and approaches to prevention and Management. Drugs 2001;61:2193-205.

3. Kranke P, Eberhart LH, Roewer N, Tramer MR. Single dose parenteral pharmacological interventions for the prevention of postoperative shivering: A Quantitative Systematic Review of Randomized Controlled Trials. Anesth Analg 2004;99:718-27.

4. Filos KS, Goudas LC, Patroni O, Polyzou V. Hemodynamic and analgesic profile after intrathecal clonidine in humans. A dose-response study. Anesthesiology 1994;81:591-601.

5. Anne Miu Han Chan, Kwok Fu. Control of shivering under regional anaesthesia in obstetric patients with Tramadol. Can J Anaesth 1999;46(3):253-8.

6. Chaturvedi S, Domkondwar G. Control of shivering under regional anaesthesia using Tramadol. Asian Archives of Anaesthesiology and Resuscitation 2002;57:491-6. 
7. Zhang Y, Wong KC. Anaesthesia and postoperative shivering: its etiology, treatment and prevention. Acta Anaesthesiol Sin 1999; 37:115-120

8. Mathews S, Al Mulla A, Varghese PK, Radim K, Mumtaz S. Post-anaesthetic shivering: A new look at tramadol. Anaesthesia 2002; 57:387-403.

9. Delaunay L, Bonnet F, Liu N, Beydon L, Catoire P, Sessler DI. Clonidine comparably decreases the thermoregulatory threshold for vasoconstriction and shivering in humans. Anesthesiology 1993; 79: 470-4.

10. Maheshwari BS, Shah SK, Chadha IA. Tramadol and butrophanol for control of shivering: randomised double blind comparative study. $J$ Anaesth Clin Pharmacol. 2008;24:343-346.

11. Atashkhoyi S, Negargar S. Effect of tramadol for prevention of shivering after spinal anaesthesia for cesarean section. Research Journal of Biological Sciences. 2008;3:1365-1369.

12. Dhimar AA, Patel MG, Swadian VN. Tramadol for control of shivering: comparison with Pethidine. Indian $J$ Anaesthesia. 2007;51:28-31.

13. Bhatnagar S, Saxena A, Kannan TR, Punj J, Panigrahi M, Mishra S. Tramadol for postoperative shivering: a double-blind comparison with pethidine. Anaesth Intensive Care. 2001;29(2):149-154.

14. Schwarzkopf KR, Hoff H, Hartmann M, Fritz HG. A comparison between meperidine, clonidine and urapidil in the treatment of postanesthetic shivering. Anesth Analg. 2001;92:257-260.

15. Horn EP, Werner C, Sessler DI, Steinfath M, Esch JS. Late intraoperative clonidine administration prevents postanesthetic shivering after total intravenous or volatile anesthesia. Anesth Analg. 1997;84:613617.
16. Chen SC, Tang CS, Chen YT, Ko CJ, The evaluation of the anti-shivering effect of tramadol during epidural anesthesia. Gaoxiong Yi Xue Ke Xue Za Zhi. 1994 Nov;10(11):632-9.

17. Parvin Sajedi, Gholamreza Khalili, Liela Kyhanifard. Minimum effective dose of Tramadol in the treatment of postanesthetic shivering. Journal of Research in Medical Sciences March \& April 2008; 13(2);75-79.

18. Zahedi H. Comparison of tramadol and pethidine for Post-anesthetic shivering in elective cataract surgery. J Res Med Sci. 2004; 5:235-9.

19. Vogelsang J, Hayes SR. Butorphanol tartrate (stadol) relives postanesthesia shaking more effectively than meperidine (Demerol) or morphine. J Post Anesth Nurs. 1992 Apr;7(2):94-100.

20. Gangopadhyay S, Gupta K, Acharjee S, Nayak SK, Dawn S, Piplai G. Ketamine, tramadol and pethidine in prophylaxis of shivering during spinal anaesthesia. $J$ Anaesth Clin Pharmacol. 2010;26(1):5963.

21. Dewitt J, Deloo FT, Deveylder J, Housmans PR. Tramadol in treatment of post anesthetic shivering. Acta Anesthesia Scand. 1997;41:506-510. 